\title{
Tendencias hacia un cine medioambiental. Concientización de una producción y diseño sustentable
}

\section{Mastantuono, Laura [ver currículum del autor, docente de la Facultad de Diseño y Comunicación]}

\section{Resumen:}

El espacio audiovisual se ha permeado y ha alcanzado las teorías del diseño sustentable que predominan en las discusiones y tendencias de la segunda década del siglo XXI. Las producciones de ficción y documental con un contenido medioambiental comienzan a ocupar lugar en los festivales de primera categoría, como así también, se presenta una tendencia emergente en la creación de algunos dedicados exclusivamente a este tipo de temática. Surge una dicotomía al observar el intento de concientización sobre el problema de la sustentabilidad, pero no la aplicación o análisis desde una perspectiva industrial. Este ensayo pretende proporcionar una articulación entre ventanas de producción y exhibición del cine medioambiental con el objetivo de comprender sus posibilidades cinematográficas.

Palabras clave:

desarrollo sustentable - diseño sustentable - producción audiovisual - protección del medio ambiente - gestión cultural - géneros.

La contaminación es la consecuencia de la introducción de un elemento ajeno al medio en el que se inserta, resultando en la alteración del mismo. La creciente preocupación y conceptualización de la contaminación desde una perspectiva histórica fue analizada en la década de 1970 cuando ideologías inherentes al contexto social, político y cultural de esa época, comenzaron a interesarse por los efectos producidos a nivel ambiental, principalmente, por las prácticas industriales. Existen dos aristas de la contaminación audiovisual, una referida a la sociedad del espectáculo y la era de la proliferación de pantallas introducidas en la vida cotidiana. La otra, 
remite a las consecuencias ambientales resultantes de la industria audiovisual en sí; este segundo ángulo es el que compete para poder analizar las prácticas de realización audiovisual sustentables. La última década del siglo XX, fue atravesada por la era de la información; el calentamiento global y el efecto invernadero se tornaron temáticas trascendentales en los distintos niveles de educación y los medios de comunicación. La creación de un cine medioambiental en vías de consolidación cómo una temática repetida estéticamente, logró atravesar los circuitos alternativos de exhibición, hacia aquellos ya establecidos -como por ejemplo festivales de primera línea- a tal punto que, inclusive, comenzaron a crearse festivales orientados únicamente a esta temática. Al mismo tiempo, la industria también demostró cierto interés hacia una realización sustentable. Las temáticas, principalmente del género documental, han virado de proyectos meramente de denuncia a documentales observacionales con cierta tendencia a un final esperanzador y/o presentando posibles soluciones. El panorama audiovisual referido a esta problemática, se presenta entonces, con cierta riqueza discursiva y productiva surcada por una dualidad antitética, la diferencia entre lo que se comunica y cómo se crea el mensaje.

\section{Ecología Racional y Factual}

"La mecánica de las modas -escribía-, de todas las modas, es bien conocida. Se toma un tema, se lo celebra durante algunos meses, e inmediatamente después de lo deja de lado, se lo fosiliza. En otras palabras, se lo declara pasado de moda". Volatilización mediante difusión, depreciación mediante dispersión. La mejor manera de sustraer rápidamente un argumento de la atención pública (o al menos restarle interés) es obligando a la gente a ocuparse del mismo insistentemente. Hoy, la moda ecológica nos está ofreciendo un ejemplo más que evidente de esta mecánica. Llevado a un grado máximo de ebullición propagandística, comienza ahora a desdibujarse, y la estridencia de las rotativas puestas a su servicio no la está haciendo más audible. Dentro de no mucho habrá cumplido si ciclo vital: una exterioridad que para nosotros era motivo de lacerante preocupación quedará definitivamente interiorizada. No se hablará más de ella. No existirá. (Maldonado, 1999. p. 13)

Se refiere a la explotación de tendencias en el mundo del diseño y la comunicación, aplicado al contacto sucedido a finales del siglo XX; dónde la repetición temática ficcional y no ficcional sobre las consecuencias que estaba sufriendo el medio ambiente, debido a prácticas no sustentables, se convertían en una tendencia discursiva. El autor, contemporáneo a la misma, considera su propia prognosis (generada en la década del '70) pesimista. El interés en rescatar este tipo de opinión funciona en tanto, en la actualidad, transcurrida más de la mitad de la segunda década del siglo XXI, pensar en la ecología cómo moda no puede estar más lejos de una aproximación errónea. La preocupación sobre las prácticas ecológicas se dividen dentro de la producción audiovisual, entre la comunicación emprendida por los medios y el diseño sustentable. La realización audiovisual posee una gran influencia en el imaginario colectivo de las audiencias sobre una gran diversidad de temas, en cuanto a la problemática que compete, es uno de los medios que logra crear empatía con el espectador de una manera contundente, por lo que resulta fácil omitir lo que sucede detrás de escena. Inclusive más factible es ignorar el impacto ambiental de las filmaciones.

Así, se presenta una dicotomía en la producción audiovisual cuando se observa la cantidad de proyectos de esta temática ante la falta de datos y hechos sobre prácticas sustentables en la realización de los mismos.

Maldonado afirma "El problema crucial que concierne a la dificultad, siempre mayor, de transferir la temática del medio ambiente desde un nivel de complejidad a otro del sistema social" (1999. p. 15). Discurriendo sobre la realidad de la dualidad antitética antes descripta, ¿cómo se traslada la preocupación por dicha problemática hacia la cotidianeidad del sistema productivo audiovisual? Maldonado toma prestado la conceptualización de los 
niveles sociales de Karl Otto Apel para poder comprender cómo afecta una temática en diversos contextos sociales. Las tres variables son la macrosfera (nivel global), mesosfera (política cultural regional) y microsfera (ambiente familiar y comunidad) (1999, p. 15). Si se re categoriza este planteamiento, se referiría a una perspectiva general cómo la totalidad de la producción audiovisual en el mundo, más acotada como la industria o producciones nacionales, también conocidas cómo cinematografías locales, finalizando en la realidad de cada producción o productora. De esta manera, se obtiene respectivamente: la macrosfera, mesosfera y microsfera audiovisual. Es sorprendente, de algún modo, la falta de datos estadísticos y verificados a nivel global, sobre las consecuencias de los desechos fílmicos. Desde un punto de vista histórico no se puede ignorar los efectos causados por la fabricación y basura generada por los soportes fílmicos analógicos (plásticos sintéticos): por una lado los ópticos, nitrato de celulosa, acetato, diacetato y triacetato de celulosa, y finalmente el poliéster. Por otro lado los soportes magnéticos. Sin embargo, debido al costo de la materia prima, la utilización de los mismos era extremadamente racional y responsable. Los costos se vieron reducidos considerablemente con el advenimiento de la era digital, en un principio se podría pensar que la misma podría colaborar con la ecología y disminución de desechos y utilización de energía. No obstante, el avance tecnológico constante y la necesidad itinerante de la producción para mantenerse continuamente actualizada generaron otro tipo de basura, la basura electrónica, la cuál es relativamente nueva y sus secuelas están siendo analizadas, no obstante, este tipo de desperdicio no puede ser comparado ante los porcentajes de emisiones peligrosas y desechos tóxicos causados por la industria petrolera, por ejemplo. (UCLA, 2006, p. 39) Desde la perspectiva de producción y los elementos utilizados en un proyecto audiovisual, ya sea cinematográfico o televisivo, se deben tener en cuenta una determinada cantidad de variables: aquellas involucradas en la preproducción, cómo utilización de papel; en el rodaje, creación de decorados, vestuario, y lo que compete a la dirección de arte. Los generadores de energía para alimentar los artefactos de la dirección de fotografía, la maquinaria involucrada en sí y materiales de grabación. Finalmente aquello involucrado en la postproducción, recursos tecnológicos y dónde se realiza el almacenamiento de datos. Por último lo pertinente a movilidad y catering, que se encuentra presente en todas las etapas. Del mismo modo, éstas son atravesadas por un tercer ángulo el del impacto que tiene la industria, la generación de basura y corrupción de los espacios dónde se filma, el uso de energía, contaminación del aire y emisión de CO2. Estas variables se deben tener en consideración para poder arribar a una conclusión sobre el accionar de las esferas audiovisuales y sus efectos adyacentes relacionados con la macrosfera. La falta de casos de estudio e investigaciones fehacientes, inclusive meros sondeos, dificulta dicha aproximación. La transcendencia del estudio de la industria audiovisual es complicada en tanto industria, cómo su significado lo indica, solamente puede ser considerada como tal en dos países Estados Unidos de América e India, con los asentamientos productivos de Hollywood y Bollywood respectivamente. Por industria se entiende a una determinada actividad económica, con su técnica pertinente y establecida, cuya generación de productos en un determinado período de tiempo alcanza un número aproximado que permite su continuidad en el tiempo, funcionando bajo las reglas del mercado. Exceptuando los países mencionados es controversial poder adjudicarle el título industrial al resto de las cinematografías locales. Entonces, se vislumbran las razones por las cuales emprender una investigación y clarificar datos a nivel global es una tarea complicada, cuando en la gran mayoría de los casos, la realización audiovisual recae en pequeñas o grandes empresas, de carácter independiente. Por lo que -en cuanto se refiere a cine independiente- no remite a un estilo discursivo sino a uno productivo. En su defecto, en un nivel más acotado, por las razones relacionadas con el problema industrial, se encuentran ciertos estudios que pueden ser tomados cómo punto de partida y de comparación para la reflexión sobre la mesosfera. En el 2006 la Universidad de Los Ángeles (UCLA) publicó un reporte Sustainability in the Motion Picture Industry, (Sustentabilidad en la Industria Audiovisual) que más de diez años después, es una de las pocas publicaciones 
académicas, que reúne datos obtenidos por medio de entrevistas e investigaciones de artículos especializados y bibliografía, creando un marco teórico sobre la huella de carbono originada por Hollywood. Según el reporte se estimó que la industria fílmica y televisiva en Los Ángeles son los responsables de la emisión de 8,4 millones de toneladas de dióxido de carbono, lo que equivale a un poco más del $25 \%$ de la causada por la Industria petrolera (p. 15). Debido al incremento en las tarifas de energía y combustibles en la primera década del siglo XX se han vislumbrado esfuerzos, por parte de los grandes estudios, para establecer estándares de un uso responsable, sin embargo dichos esfuerzos y cierta concientización sobre el tema ya data de los años 90, cuando por ejemplo la producción de Matrix (Wachovwsky, 1999) reutilizó y recicló los decorados que fueron creados, o la serie televisiva The X-Files (Carter, 1993), de la cadena FOX, planteó un esbozo de movilidad sustentable. Tanto este estudio como Sony declaran tener políticas sustentables en la realización de sus productos. Ambas compañías poseen páginas web dedicadas a la comunicación de los esfuerzos realizados para gestionar acciones efectivas en la protección del medio ambiente y controlar la contaminación generada. De esta manera, se aprecia una concientización en el medio y la forma de operar que poseen, como por ejemplo, la creación de sistemas de energía solar para poder alimentar los distintos departamentos en todas las etapas productivas. Sin embargo, sin una regulación a escala nacional los esfuerzos se deben considerar a un nivel, de lo que antes se mencionó cómo microsfera. La microsfera es el eslabón más pequeño de estas categorías re conceptualizadas para el medio audiovisual. Aquella que se refiere a las productoras y proyectos. Uno de los inconvenientes dados en este escalafón es el grado de compromiso tomado por compañías que luchan por poder realizar sus proyectos o tiene un corto período de vida, en lo que especta al cine independiente. Lo mismo sucede en la cadena industrial al reflexionar sobre las pequeñas productoras absorbidas por las grandes compañías. La intención de compromiso se ve sesgada por el presupuesto del producto realizado, y si bien aquellos con recursos acotados tienen un impacto menor en los niveles de contaminación a gran escala, éste debería ser analizado por una concientización establecida en las consecuencias que involucran la realización audiovisual y no una casualidad. Existen muestras que dejan asentado un modelo a desarrollar y seguir. Se plantea reflexionar sobre la cuestión por medio de tres casos que pueden ilustrar la voluntad y concientización que se está generando en el medio: una serie televisiva que en todo su recorrido plantea el problema medio ambiental contadas veces, un largometraje ficcional cuyo contexto se basa en el calentamiento global y un largometraje documental cuyo recorrido argumental se focaliza en la relación del ser humano con su alrededor. The X-Files, además de apuntar a una comunicación transparente a través de la página online de la cadena, dedicada a transmitir el impacto social que producen, también declaró poseer una mediación y gestión ecológica por medio de ciertas prácticas sustentables, cómo la utilización de combustibles alternativos y reciclaje de decorados. La producción de la serie, que regresó al aire luego de 11 años, (considerando la segunda película como parte de la instalación narrativa de la serie) manifestó querer continuar con las prácticas sustentables con las que trabajaron en la década de 1990 y principios de siglo; por lo que el estudio les proporcionó una consultoría que se dedicó a auxiliar en cada área y departamento creativo. Gracias a estos esfuerzos la producción logró reciclar el total del hierro y aluminio utilizado en la construcción de decorados, y re direccionar el $81 \%$ de la basura generada para relleno en construcciones. A su vez, algo tan simple cómo reemplazar botellas descartables de agua significó evitar desperdiciar más de 45 mil de estos envases. (21st Century Fox, 2016) El día después de mañana (Emmerich, 2004) es otro caso que merece ser mencionado. La película, un largometraje apocalíptico de ciencia ficción, describe un cambio abrupto y extremo en el clima asociado con el calentamiento global. Para la misma no sólo se necesitaba una gran cantidad de efectos especiales en escena sino también en post producción, lo que generaría un impacto ambiental aún mayor que el promedio de las producciones. Sin embargo, el director comprometido con la temática en la cual se basaba su proyecto, contacto a la organización medio ambiental 
Future Forests. La misma se encarga de plantar árboles para contrarrestar la emisión de dióxido de carbono. La compañía estimo las emisiones de la película en 10 mil toneladas de $\mathrm{CO} 2$ y recibió el monto necesario para la forestación, de esta manera neutralizando la huella de carbono. (UCLA, 2006, p. 36) Estos casos que ilustran el panorama norteamericano, dan lugar al cuestionamiento sobre el estado de la cuestión en el resto del mundo. A nivel nacional o regional no pueden encontrase datos fehacientes que den cuenta de la concientización del problema. Bollywood, recién en el año 2015 generó una producción con una huella de carbono neutra. La problemática sobre el impacto ambiental generado por la producción audiovisual acarrea entonces otro conflicto, la falta de publicaciones, especializadas y no especializadas, sobre el tema. Se realizó una división teórica entre las producciones industriales e independientes, no obstante, también se deben diferenciar las consecuencias de la realización de productos ficcionales y documentales, ya que ciertos elementos, como la creación de decorados, no son característicos del documental. La movilidad y consumo energético y de materias primas siguen siendo variables a analizar. La sal de la tierra (Salgado y Wenders, 2014) es un largometraje documental sobre la vida y obra del fotógrafo Sebastião Salgado, denuncia las migraciones por el accionar político y abuso medioambiental del ser humano. El mismo da un vuelco cuando comienza a narrar el esfuerzo realizado por el protagonista y su familia, al regresar a su ciudad de origen, Aimorés, en Brasil. El cambio climático en la región, debido a décadas de deforestación es contundente, observando una sequía devastadora dónde antes había tierras fértiles y proliferaba la diversidad biológica. En 1998 la familia Salgado funda el Instituto Terra con el plan de forestar de nuevo la zona, y en 15 años se logra regresar al clima nativo de la zona. La experiencia documentada presenta una solución a un problema medioambiental, la falta de agua, ya que con la fertilidad de las tierras y ecosistemas característicos de los pastizales y bosques, también desaparece la sequía. Si bien la película no tiene datos estimativos del impacto ambiental que pudo haber producido, al haber reforestado más de 17 mil hectáreas, se puede arriesgar que la huella de carbono de la misma, también fue neutralizada. Los casos convergen en que todos han logrado una unión interdisciplinaria, con organizaciones por fuera de la realización audiovisual. Por lo tanto, se puede arribar a la reflexión sobre la necesidad de fomentar estudios desde un nivel regional o estatal, para así poder aglomerar información a nivel mundial. Si bien los ejemplos mencionados son sólo algunos de los que uno encuentra a nivel de la microsfera, se necesita una concientización a mayor escala, así como también determinadas reglas.

\section{Comunicación y consumo, hacia una cultura ecológica}

Desde que la contaminación y su conceptualización se asimiló en la vida cotidiana del ser humano, el tipo de discurso presente en los medios de comunicación evolucionó, al igual que la aproximación del hombre y la relación con su alrededor. Los efectos de décadas de abuso inconsciente del entorno han dado lugar a pequeños cambios en el estilo de vida del ciudadano privilegiado, mientras que la brecha con aquellos que no poseen acceso a las necesidades básicas aumenta. En un principio los medios se encargaron de proporcionar información generalizada sobre las variaciones en el contexto medioambiental a nivel global, una comunicación tendenciosa privilegiando noticias sobre hechos que factiblemente pueden tener una solución e ignorando otros, sin embargo el discurso contemporáneo deja de lado el egoísmo característico del antropocentrismo y da cuenta de pequeños sucesos y las posibles medidas a tomar.

No se puede oponer abstractamente el espectáculo y la actividad social efectiva. Este desdoblamiento se desdobla a su vez. El espectáculo que invierte lo real se produce efectivamente. Al mismo tiempo la realidad vivida es materialmente invadida por la contemplación del espectáculo, y reproduce en sí misma el orden espectacular concediéndole una adhesión positiva. La realidad objetiva está presente en ambos lados. Cada 
noción así fijada no tiene otro fondo que su paso a lo opuesto: la realidad surge en el espectáculo, y el espectáculo es real. Esta alienación recíproca es la esencia y el sostén de la sociedad existente (Debord, 2008, p. 33)

El cine, espectacular o de ensayo, ha evolucionado como reflejo de la realidad, ya sea en un primero o segundo plano de análisis. Los temas presentes, cómo la multiplicación de historias dentro del código de determinados géneros, ocurrieron siempre en respuesta del contexto social, económico y político contemporáneo a la realización. La creación artística no es arbitraria, del mismo modo que la tecnología responde a cambios en las necesidades de la humanidad, a la misma vez que el todo revela su lazo intrínseco con el mercado. Todo concepto que se estableció en la cultura tuvo un proceso histórico surcado por el diálogo entre consumidor y productor emisor. Sería inocente reflexionar sobre el problema ecológico ignorando el contexto en el que fue dado, evitar el cuestionamiento de la causa y efecto. De este modo, la variación del discurso ocurre debido a la democratización de la generación de información y el acceso a la misma. Por otro lado consecuentemente la notabilidad sobre el tema permeó diferentes estratos del contexto social de acuerdo al surgimiento de necesidades y planteamientos teóricos y prácticos. Finalmente, se reparó en los recursos naturales cómo agua potable, aire puro, bosques nativos e implantados, pastizales, la biodiversidad marina y terrestre de acuerdo a las variaciones climáticas del planeta; la necesidad de salvaguardar dichos recursos, elude los límites políticos y naturales, esta premisa comenzó no sólo a penetrar en el imaginario colectivo lentamente, sino a invadir el mensaje creado por el discurso cinematográfico medioambiental. Las consecuencias del accionar del hombre, sin una reflexión crítica sobre él mismo, fueron descriptas y narradas, metafóricamente o no, fundamentalmente en el género de ciencia ficción. Primero en la literatura y luego en la cinematografía. Antes de que existieran reglas que estandarizaran el discurso audiovisual, se pueden encontrar películas ficcionales con temáticas que favorecen un mensaje crítico sobre la posible dirección del mundo si el contexto social no daba cuenta de los efectos de su proceder. Este tipo de producciones se centraban principalmente en mundos apocalípticos futuristas, y este tipo de historias perduraron hasta la postmodernidad, cohabitando en las esferas audiovisuales con documentales de denuncia que acercan información sobre comunidades que no son cercanas al espectador promedio, como por ejemplo el largometraje, Virunga (von Einsiedel, 2014). Producido en asociación con Netflix, trae a colación otra cara de la industria audiovisual, la de las plataformas digitales. El documental, luego de haber realizado su recorrido por festivales, y premiaciones fue distribuido por la plataforma online alrededor del mundo, dando a conocer el estado de la cuestión en el Parque Nacional que da nombre a la pelí- cula. Narra la lucha de los guardaparques por preservar el lugar y hábitat de los últimos gorilas de montaña existentes en el mundo, mientras que son amenazados por la guerra en la República Democrática del Congo, sus consecuentes migraciones, impacto ambiental generado por las actividades bélicas y la posible explotación petrolera. La denuncia plasmada en el documental alertó globalmente, de tal manera, que la compañía intentando penetrar en el Parque para extraer combustibles, se retiró. Este tipo de discurso perdura en el tiempo pero logra, por medio de la proliferación de pantallas y acceso a la información, propagar la concientización de otra manera, dando lugar a una nueva tendencia de discursos esperanzadores, dónde no solo se alerta sobre el estado de la cuestión sino que también se presentan posibles soluciones, dando lugar a la reflexión y a la acción de una mediación medioambiental del ser humano consciente y eficaz, en la preservación de los recursos naturales presentes en el planeta. Las películas con ésta temática se observan en todos los géneros cinematográficos. Sin embargo, existe una preponderancia de algunos y de acuerdo a los mismos difiere el mensaje y las variaciones en la lectura del mismo, correspondiente a las reglas discursivas aplicadas. La categorización se puede dividir en dos grandes grupos; en una primera instancia, aquellas dónde la temática recae en el subtexto, mientas que 
el conflicto principal puedo no estar relacionado visiblemente con la misma y aquellas dónde el conflicto principal está intrínsecamente ligado al accionar irresponsable del ser humano y la asimilación del mensaje se encuentra en un primer nivel. De acuerdo a esta conceptualización por ejemplo, se puede remitir respectivamente a: Buscando a Nemo (Stanton, 2003) y La Princesa Mononoke (Miyazaki, 1999) Ambos films producidos por reconocidos estudios de animación, Pixar y el Estudio Ghibli, traen a colación la responsabilidad del hombre y la alteración que causa en su entorno. Buscando a Nemo, si bien trabaja sobre un contexto dónde el ser humano está destruyendo los arrecifes de coral y el argumento recae en los cambios conductuales de los animales como resultado de la explotación de la biodiversidad marina, el conflicto principal refiere a la relación de padres e hijos. La Princesa Mononoke, en cambio, coloca en un primer plano discursivo la destrucción de la naturaleza en las manos del hombre, narrando la historia épica de una princesa y los guardianes fantásticos de un bosque contra el abuso de los recursos naturales a mano del ser humano. Más allá de la temática también existe una evolución en la formalidad de estas narraciones, dónde la estética popular y colores estilizados presentes en las películas de denuncia, debido, en gran parte, a la utilización de material de archivo y estética televisiva; dieron paso a la estética de autor y un discurso postmoderno, al igual que las películas de otras temáticas. El cine medioambiental no puede ser analizado fuera del contexto cinematográfico mundial. El panorama actual, debido a la digitalización y sus posibilidades, cómo la accesibilidad a los equipos permitieron una proliferación de miradas. La yuxtaposición de este momento con la incipiente preocupación por el los cambios climáticos y destrucción de los recursos naturales tuvieron como consecuencia la aparición de pantallas dedicadas a esta temática y la multiplicidad de festivales dedicados a la misma. Los festivales de cine medioambiental surgieron a finales de 1980, en la década siguiente se multiplicaron alrededor del mundo, logrando crear redes en el siglo $X X I$. Son las ventanas de exhibición que suelen tomar un argumento en auge, creando un espacio de exposición; y de esta manera permiten al público acercarse a las diversas perspectivas sobre lo que sucede en el mundo contemporáneo. Sin embargo, para los críticos cinematográficos estos festivales aún son comprendidos cómo festivales jóvenes, por lo que las películas que exhiben no reciben la atención que merecerían, teniendo en consideración la necesidad de promover una gestión cultural de mediación con el medioambiente que se desarrolló a lo largo del ensayo. El crecimiento de este tipo de festivales, así como también de películas con esta temática, es una dicotomía, en tanto la industria se encuentra en un continuo esfuerzo por lograr estandarizar un diseño sustentable de producciones audiovisuales; al mismo tiempo que la academia y la crítica ignora la incipiente preocupación por cumplir un rol en la tendencia de preservación ecológica que ocurre en diversas disciplinas. Además, los festivales cumplen un rol dentro de la industria audiovisual, al ser no sólo un espacio dónde ciertas películas, que no logran un éxito comercial, son exhibidas, sino también un lugar dónde realizadores y público se encuentran y ocurren conexiones, no sólo profesionales sino también relacionadas con el tema, problema y posible solución planteados en cada film. Funcionan cómo un fenómeno en sí mismos, dando oportunidad a una identificación cultural de la comunidad. Los festivales, además, destacan las prácticas medioambientales que toman, dejando de lado el envío en papel, ahorrando energía y materia prima, fomentan la distribución digital, y las prácticas que el público puede llevar a cabo en la cotidianeidad cómo la reducción de basura y envases reciclables. A su vez, comúnmente presentan stands dónde el público puede acercarse y conocer sobre el accionar de ciertas Organizaciones No Gubernamentales o los colectivos que produjeron las películas exhibidas, de esta manera involucrándolos en el proceso de preservación. No se puede tener una mirada inocente hacia el accionar de los festivales por lo que la misión de cada uno que se comunican en sus sitios web debe ser analizada antes del envío del material. En general, el objetivo de los mismos es poder generar la reflexión individual sobre el rol que cumple cada uno en el cuidado de la biodiversidad y como se puede proceder a un accionar en comunidad. Los festivales además, cumplen una 
función en el mercado, la de poder vender y distribuir las películas. En la Argentina la medición de la huella de carbono en el ámbito cinematográfico es una conversación que todavía tiene un largo proceso de investigación y promoción por resolver. La cantidad de artículos encontrados que tratan sobre el tema son escasos, si bien en el año 2015, la comunicación del Green Film Fest, el festival cinematográfico medioambiental más reconocido a nivel nacional, prometió evaluar el impacto ambiental del evento para poder encontrar soluciones y neutralizar la emisión de carbono producida. Lo mismo sucede en Canadá, por ejemplo, donde el Festival Planet in Focus, pretende achicar la brecha entre los esfuerzos y el accionar, uniéndose con compañías que llevan a cabo la neutralización de la huella de carbono en la industria audiovisual, determinando los datos del impacto generado, como así también, la energía ahorrada en estas producciones y el festival en sí (Armatage, 2013, p. 259). Los festivales no solo funcionan como ventanas y agentes de información sobre el problema competente, además, proporcionan soluciones, una comunicación clara y precisa que a través de ciertas pautas atrae a la acción de profesionales y no profesionales. La representación que generan estos festivales, ya sean de grandes organizaciones y compañías productivas o de un cine alternativo, se encuentra entre medio de lo que es el panorama heterogéneo de la mercantilización de los medios, quedando a la vista la lucha de los realizadores independientes y de la gestión cultural en sí, para consolidarse en este territorio. Es una necesidad imperante, el fortalecimiento entre este nicho de exhibición y punto de encuentro entre el discurso y la producción para poder sentar las bases y modelos de un código de preservación ecológica por parte de los sujetos involucrados en la realización audiovisual.

Hacía un código y la acción

El espacio audiovisual se ha permeado y aproximado a las teorías del diseño sustentable que predominan en las discusiones y tendencias de la segunda década del siglo XXI. Las producciones de ficción y documental con un contenido medioambiental comenzaron a ocupar lugar en los festivales de primera categoría, cómo es el caso de Virunga y La Sal de La Tierra. A la vez que los festivales dedicados exclusivamente a este tipo de temática encuentran un nicho y se estabilizan en el panorama audiovisual. Una de las redes consolidadas de este tipo de actividad, Green Film Network, reúne los festivales más importantes de cada país y algunos regionales; sin embargo no todos figuran en la misma, y aquel realizador (independiente) que decidió producir una película cuya trama está atravesada por dicha problemática, tiene que embarcarse en una investigación profunda para poder lograr encontrar el target para su producto. Independientemente de la investigación característica para poder narrar una historia de este tipo, ya que es necesario colocar en tela de juicio la responsabilidad ética de los realizadores, concernida con la comprensión del punto de vista de la realidad narrada. La experiencia propia dicta que si bien es una tarea exhaustiva existen posibilidades y es factible de concretar alrededor del mundo. El abanico de ventanas de exhibición, tradicionales y las cuáles se originaron luego del traspaso al digital y plataformas online, abren el camino para alcanzar el objetivo de que la película, cortometraje, mediometraje o largometraje, arribe a las audiencias. A la vez, es importante que, desde las microsferas audiovisuales, continúen los esfuerzos por promover las producciones sustentables. Por medio del acercamiento a corporaciones privadas o estatales lograr mitigar la huella de carbono y reducir los desechos es factible. Sin embargo, el estudio sobre el impacto generado por la realización audiovisual también es necesario. Hay una pretensión en la articulación de la misión de festivales de cine medioambientales y el cómo se produce. La misma ha crecido en la segunda década del siglo XXI, logrando abordar una gestión cultural de la mediación ambiental. Además de lo concerniente al aspecto profesional, también relacionado con poder inspirar y que el espectador haga una diferencia en su día a día. Más allá de la voluntad individual los casos observados 
concuerdan en lo referente a un lazo interdisciplinario, uniones que permitan concretar la preservación medio ambiental y por medio de esta articulación lograr dar cuenta de la necesidad de establecer una cultura que reflexione sobre la relación ser humano - naturaleza. El marco teórico del ensayo hace hincapié en la creación de un código y jurisprudencia sobre el accionar de la producción audiovisual para la aproximación a la sustentabilidad a nivel de la macrosfera. Analizar la coherencia entre el contenido generado para las audiencias y la educación del profesional del medio.

Se nos anda olvidando que respiramos el alma verde los árboles. La transparencia que nos anima por dentro es, en efecto, la tarea lograda por los bosques. Cada árbol es una fuente y un diminuto clima local. Plantar árboles es además cultura porque los bosques han publicado todos los libros. Porque casi todos los campos cultivados fueron tierras de arboleda. Porque la palabra agricultura fue la que fundó el término cultura.

O, mejor aún, sobre los suelos que primero construyó el árbol luego los humanos crearon civilizaciones. (Araujo, 2014, p. 60)

La deforestación y reforestación son dos de los puntos más reconocidos o posibles de comprender en la lucha de la preservación medioambiental. Son los reconocidos pulmones del planeta y el elemento visual por excelencia en la educación ecológica. A la misma vez, son aquellos que logran crear una incidencia en el proceso biológico estudiado para reducir el impacto del carbono en el medioambiente y sus consecuencias. De acuerdo a los reportes analizados a lo largo del trabajo se vislumbra en primera instancia realizar el debido cálculo de la emisión de carbono de cada producción. De esta manera, el ahorro de energía y el aporte necesario para poder neutralizar el mismo es viable. Apuntar hacia la creación de un código ético laboral que funcione como un diálogo entre lo que se comunica y cómo se procede. La realización del mismo necesita de terminadas etapas: crear un protocolo de medición del impacto general que realiza la compañía o producción, no sólo de carbono. Los principios para calcular las emisiones deberían ser consultados con otras disciplinas. Por este motivo, es importante la estandarización de reglas a nivel global. No obstante reducir las emisiones contaminantes se consigue por medio de un consumo y marketing responsable. Se pueden recortar utilizando envases y vajilla re utilizables para generar menos basura. La comunicación dentro del equipo también es trascendental, atrayendo ideas que posiblemente sean utilizadas en la vida cotidiana, del mismo modo que la estrategia de movilidad afecta el impacto total. Asimismo, se deben repensar las estrategias de marketing y prensa de cada película por medio del uso consciente de las materias primas que dañan el ecosistema. Poder eliminar la huella de carbono por completo no es posible, pero sí lo es la compensación, cómo se vio en los casos del Día después de mañana o La Sal de la Tierra, pero también se puede lograr a través de la instauración de combustibles sustentables y energía renovable como paneles solares o molinos de viento. Es anacrónico pensar en dejar de lado completamente el estilo de vida y de producción del contexto post moderno, bajo una bandera de preservación medioambiental. Sin embargo, es necesario poder dejar de dar por sentado los recursos naturales presentes en el planeta para poder adquirir esa mirada crítica hacia lo que le es narrado, la información recibida, y la información creada a través de la observación empírica y personal. Las posibilidades se encuentran en la búsqueda de un diálogo que permita una transformación del consumo irresponsable hacia el empleo de los recursos de manera reflexiva y crítica. Proceso que puede ser observado a través de las respuestas y alfabetización del espectador promedio, el llamado prosumidor. Ya no existe una audiencia maravillada por la mera narración de la imagen en movimiento y espectacularización de la misma, son distinguibles diversos marcos de recepción y la capacidad de evaluación del público postmoderno, la evolución del discurso audiovisual ocurrió de la mano de la educación del consumidor. Es por esto, que la creación de un 
código unificado entre las diferentes etapas en la realización audiovisual, si bien podría ser considerado una utopía, apunta a la clarificación de una retórica coherente. Si no, el intento de concientización, sin el accionar propio, pierde sinceridad.

\section{Referencias Bibliográficas}

21st Century Fox (2016). Take an Exclusive Look Behind the Scenes of 'The X-Files' and Learn How Fox Went Green. Recuperado el 17/02/2016 de https://impact.21cf.com/ what/2016/02/x-files-green-production.html Araujo, J. (2014). Epílogo. En Gionno, J. El hombre que plantaba árboles (pp. 60-62). Barcelona: Duodécimo Ediciones. Armatage, K. (2013). Planet in Focus: Environmental Film Festivals. En Pick, A. y Narraway, G. (Eds.). Screening Nature: Cinema beyond the Human (pp. 257-273). Nueva York: Berghahn. Buchanan, A. y Engel, L. (2009). Code of Best Practices in Sustainable Filmmaking. Washington DC: American University, School of Communication. Disponible en: http://cmsimpact. org/sites/default/files/final_code.pdf Carter, C. (Productor) (1993). The X-Files. [Serie de televisión] FOX. Debord, G. (2008). La sociedad del espectáculo. Buenos Aires: La Marca Editora. Emmerich, J. (Director) (2004). El día después de mañana. [DVD] Gativideo. Maldonado, T. (1999). Hacia una racionalidad ecológica. Buenos Aires: Infinito. Miyazaki, H. (Director) (1999). La Princesa Mononoke. [DVD] Dimension Films. Salgado, J. (Director) y Wenders, W. (Director y Productor) (2014). La Sal de la tierra. [Cine] LAT-E. Stanton, A. y Unkrich, L. (Directores) Lasseter, J. (Productor) (2003). Buscando a Nemo. [DVD] Gativideo. Times of India (2015). Bollywood should go green, reduce carbon footprint. Recuperado el 22/02/2016 de: http://timesofindia.indiatimes.com/good-governance/west-bengal/ Bollywood-should-go-greenreduce-carbon-footprint/articleshow/48395717.cms University of California Los Angeles, Institute of the Enviroment (2006). Sustainability in the Motion Picture. Disponible en:

http://www.environment.ucla.edu/perch/resources/mpisreport.pdf Von Einsiedel (Director) y Di Caprio (Productor) (2014). Virunga. [Online] Netflix. Wachowsky, A. y Wachosky, L. (Directores y Productores) (1999). Matrix. [DVD] Argentina Home Video.

\section{Abstract:}

The audiovisual space has permeated and has achieved sustainable design theories that dominate discussions and trends in the second decade of the 21 st century. The productions of fictional and documentary films with an environmental content begin to occupy a relevant place in top festivals. Likewise, an emerging trend is shown in the creation of some films dedicated exclusively to this genre. A dichotomy arises when observing the attempt to create awareness about the problem of sustainability, but not the application or analysis from an industry perspective. This paper aims to provide a link between industrial production and environmental film in order to understand their cinematographic possibilities.

Key words:

sustainable development - sustainable design - audiovisual production - environment protection - cultural management - genres.

Resumo: 
O espaço audiovisual foi permeado e alcançou as teorias do design sustentável que predominam nas discussões e tendências da segunda década do século XXI. As produções de ficção e documental com um conteúdo de meio ambiente começam a ocupar lugar nos festivais de primeira categoria. Também se apresenta uma tendência emergente na criação de alguns dedicados exclusivamente a este tipo de temáticas. Surge uma dicotomia ao observar o intento de conscientização sobre o problema da sustentabilidade, mas não a aplicação ou análise desde uma perspectiva industrial. Este ensaio pretende propor uma articulação entre janelas de produção e exibição do cinema meio - ambiental com o objetivo de compreender suas possibilidades cinematográficas.

Palavras chave:

desenvolvimento sustentável - design sustentável - produção audiovisual - proteção do meio ambiente - gestão cultural - gêneros.

Tendencias hacia un cine medioambiental. Concientización de una producción y diseño sustentable fue publicado de la página 123 a página135 en Cuadernos del Centro de Estudios de Diseño y Comunicación № 64 